\title{
Finite size corrections within the continuum limit for quantum spins: two-magnon bound states in 1D Heisenberg ferromagnet
}

\author{
A. Ceulemans ${ }^{\mathrm{a}}$, S. Cojocaru ${ }^{\mathrm{b}}$, and L.F. Chibotaru \\ Division of Quantum Chemistry, University of Leuven, Celestijnenlaan 200F, 3001 Leuven, Belgium
}

Received 28 May 2000 and Received in final form 6 April 2001

\begin{abstract}
A continuum medium approach is proposed to describe the finite size dependent effects for the 1D isotropic Heisenberg ferromagnet. The results are compared to the exact Bethe ansatz solution for the finite chain. The approach is shown to adequately account for the behaviour of the eigenfunctions and eigenenergies. The continuum is obtained by integration in Fourier space via introduction of cut-offs at the integration limits and analytical continuation from the discrete lattice to the continuous medium. It offers a new perspective on the instability of bound states, and reveals the linear behaviour of the amplitude in the critical region and other features of the model in an analytical way. We further apply this approach to investigate the long wavelength expansion of the master equation and to show the route of constructing reliable approximations valid for more complicated models. It is concluded that the approach can be useful to study mesoscopic spin systems.
\end{abstract}

PACS. 75.50.Xx Molecular magnets - 75.30.Ds Spin waves

\section{Introduction}

Design of molecular magnets as large spin clusters with specific annular or globular geometries demands a development of new theoretical models capable to accurately describe large assemblies of interacting spins distributed on a curved surface. Since the low energy excitation spectrum of such systems is characterized by a wavelength which substantially exceeds the interspin separation, these models may well be based on a continuum medium approximation of quantum spins. Analogous strategies have been already applied successfully to study electronic and vibrational structures for a wide range of polyhedral clusters $[1,2]$. The usual continuum approximation refers to the quasiclassical spin limit: $\hbar \longrightarrow 0$, $S \longrightarrow \infty$ with $\hbar S=$ const. In this limit, the quantum spin behaves as a classical vector field on the support manifold. This opens a route for applying topological analysis and for obtaining special types of solitonic solutions [3].

The premise to the present programme is quite different. The lattice is indeed smoothened to a continuous manifold, but the spins retain their quantum characteristics. We examine the possibility to incorporate the size dependent features which could be important for mesoscopic spin systems into such a continuum limit description.

In the present work we show how such a continuum approach can be developed for the one-dimensional isotropic

\footnotetext{
${ }^{a}$ e-mail: arnout.ceulemans@chem.kuleuven.ac.be

b On leave of absence from the Institute of Applied Physics, Academy str.5, Chişinău, 2028, Moldova
}

Heisenberg chain on the basis of the earlier continuum treatments $($ e.g. $[4-7])$. The analytical continuation from a discrete lattice to a continuum allows us to obtain the excitation amplitudes explicitly. Moreover, we demonstrate that such a continuum approach describes the finite size effects and, therefore, it is rather useful to study mesoscopic systems. Interestingly, we find that the long wavelength instability of the bound state which occurs at momenta $\sim 1 / \sqrt{N}$ in Bethe's finite chain solution [8] is adequately described within the present continuum approach. Thus, the $1 \mathrm{D}$ problem validates the proposed continuum treatment and the long wave approximations derived on its basis by allowing comparison to the exact solution (see $[9,10]$ for a recent discussion). The insight gained in this way can be used in treating more complicated models.

\section{Bethe's solution of the discrete problem}

Let us consider the Heisenberg Hamiltonian for a onedimensional cyclic chain of $N$ sites occupied by $S=1 / 2$ spins.

$$
\begin{aligned}
H & =-J \sum_{n=1}^{N} \mathbf{S}_{n} \cdot \mathbf{S}_{n+1} \\
& =-J \sum_{n=1}^{N}\left[\frac{1}{2}\left(S_{n}^{+} S_{n+1}^{-}+S_{n}^{-} S_{n+1}^{+}\right)+S_{n}^{z} S_{n+1}^{z}\right] .
\end{aligned}
$$

Here $S_{n}^{ \pm} \equiv S_{n}^{x} \pm \mathrm{i} S_{n}^{y}$ are spin flip operators and the interaction constant $J$ is taken to be positive for a ferromagnet. 
$H$ acts on Hilbert space of dimension $2^{N}$ spanned by the orthogonal basis vectors $\left|\sigma_{1} \ldots \sigma_{N}\right\rangle$ where $\sigma_{n}$ corresponds to spin up or spin down state at the site $n$. The state $|0\rangle \equiv|\uparrow \ldots \uparrow\rangle$ with all spins aligned in one direction is the ground state of $H$ with the eigenenergy $E_{0}=-J N / 4$. Single magnon eigenstates are given by

$$
\left|\psi_{P}\right\rangle=\frac{1}{\sqrt{N}} \sum_{n=1}^{N} \mathrm{e}^{\mathrm{i} n P} S_{n}^{-}|0\rangle,
$$

where the wavenumbers $P=2 \pi l / N(l=0, \ldots, N-1)$ are the quantum numbers of the model. To treat the two magnon excitation

$$
|\psi\rangle=\sum_{1 \leq n_{1}<n_{2} \leq N} a\left(n_{1}, n_{2}\right) S_{n_{1}}^{-} S_{n_{2}}^{-}|0\rangle
$$

Bethe [8] introduced an ansatz for the amplitude which accounts for magnon interaction through some constraints on its parameters. Additional constraints are imposed by the normalization of the wave function and the translational symmetry. The latter one is simply expressed as

$$
a\left(n_{1}, n_{2}\right)=a\left(n_{2}, n_{1}+N\right)
$$

and plays an important role in formulating the continuum limit. Furthermore, (2) implies a decoupling of the "center of mass" and relative degrees of freedom:

$$
\begin{aligned}
a\left(n_{1}, n_{2}\right) & =\frac{\exp (\mathrm{i} P R)}{\sqrt{N}} a(X) ; \\
X & =\left(n_{2}-n_{1}\right)=1, \ldots, N-1 ; \\
R & =\frac{n_{1}+n_{2}}{2} .
\end{aligned}
$$

Introducing then the auxiliary amplitude $a(0)$ results in the following master equation

$$
\begin{gathered}
{[E-2 J] a(X)+J \cos \left(\frac{P}{2}\right)(a(X+1)+a(X-1))=} \\
J\left[\cos \left(\frac{P X}{2}\right) a(0)-a(X)\right]\left(\delta_{X, 1}+\delta_{X, N-1}\right)
\end{gathered}
$$

where $\delta_{X, 1}$ is the Kronecker $\delta$-symbol.

Notice that although the nonphysical amplitude $a(0)$ exactly vanishes on both sides of equation (4), its introduction allows us to maintain the free magnon form of the l.h.s. and to consider the r.h.s. as a magnon interaction. All the solutions of equation (4) describing the subspace with two flipped spins can be divided into four classes. Two classes correspond to noninteracting objects, viz., the projections of the ground state and of the one-magnon states onto this subspace. They are characterized by the energies $J(1-\cos P)$ and the amplitude $a(X)=\cos \left(\frac{P X}{2}\right)$ where $P=0$ for the former class and $P \neq 0$ for the latter one. Indeed, it is easily seen that such an amplitude results in vanishing of the r.h.s. of (4), i.e., in the absence of interaction. On the other hand, the l.h.s. gives a free magnon dispersion. The remaining two classes correspond to the scattered and bound states which form the set of nontrivial solutions.

There exist two symmetry constraints imposed on the amplitude. The first one follows from the commutation of spin operators on different sites $\left(a\left(n_{1}, n_{2}\right)=a\left(n_{2}, n_{1}\right)\right)$ while the second one comes from the cyclic condition (2)

$$
\begin{aligned}
& a(X)=a(-X) \\
& a(X)=\exp \left(\frac{\mathrm{i} P N}{2}\right) a(N-X) .
\end{aligned}
$$

Quantization of the total momentum $P N / 2 \pi=l$ then implies the existence of two types of eigenstates with different parity, symmetric and antisymmetric, for $l$, respectively, even or odd. Equation (4) provides thus the desired platform to introduce the continuum limit. In a subsequent section we first approach this limit by a straightforward Fourier expansion of the amplitudes.

\section{Fourier expansion and the continuum limit}

Let us consider the Fourier series expansion

$$
\begin{aligned}
a(X) & =\frac{1}{N} \sum_{Q} b(Q) \exp (\mathrm{i} Q X), \\
b(Q) & =\sum_{X=0}^{N-1} a(X) \exp (-\mathrm{i} Q X) .
\end{aligned}
$$

Equations (5a) and (5b) determine the range of values $Q$ depending on the total momentum:

$$
1=\exp \left(\mathrm{i} N\left(\frac{P}{2}+Q\right)\right)=\exp \left(\mathrm{i} N\left(\frac{P}{2}-Q\right)\right) .
$$

The first equality in (7) is derived by combining the two symmetry relations

$$
\begin{aligned}
& \sum b(Q) \exp (\mathrm{i} Q X)=\sum b(Q) \exp (-\mathrm{i} Q X) \\
& =\exp \left(\mathrm{i} \frac{P N}{2}\right) \sum b(Q) \exp (\mathrm{i} Q N-\mathrm{i} Q X) .
\end{aligned}
$$

The second one is obtained in a similar way via the substitution $X \rightarrow N-X$. Consequently, the following values for $Q$ in (6)

$$
Q=\frac{2 \pi m}{N} ; \quad m=0,1, \ldots, N-1
$$

are allowed for the even parity state and

$$
Q=\frac{2 \pi m}{N}+\frac{\pi}{N} ; \quad m=0,1, \ldots, N-1
$$

for the odd parity state.

Consider first the symmetric solution. Multiplying both sides of equation (4) by $\exp (-\mathrm{i} Q X)$ and summing 
over $X$, one obtains the Fourier components:

$$
\begin{aligned}
(E / 2 J- & \left.\left(1-\cos \left(\frac{P}{2}\right) \cos Q\right)\right) b(Q)= \\
& \frac{\cos Q}{N} \sum_{Q^{\prime}} b\left(Q^{\prime}\right)\left(\cos \left(\frac{P}{2}\right)-\cos \left(Q^{\prime}\right)\right),
\end{aligned}
$$

or finally,

$$
b(Q)=C \frac{\cos (Q)}{\left[1-E / 2 J-\cos \left(\frac{P}{2}\right) \cos Q\right]},
$$

where

$$
C=-\frac{1}{N} \sum_{Q^{\prime}} b\left(Q^{\prime}\right)\left[\cos \left(\frac{P}{2}\right)-\cos Q^{\prime}\right] .
$$

This implies particularly that $b(Q)$ is an even function of $Q$, in accordance with the symmetry constraint (5a).

The compatibility condition is derived by substituting $b(Q)$ into $C$, viz.,

$$
1=\frac{1}{N} \sum_{Q^{\prime}} \frac{\cos \left(Q^{\prime}\right)\left[\cos \left(\frac{P}{2}\right)-\cos Q^{\prime}\right]}{\left[E / 2 J-1+\cos \left(\frac{P}{2}\right) \cos Q^{\prime}\right]} .
$$

It determines the energy $E_{s}$ of the symmetric bound state lying below the two-magnon continuum, $E<E_{L}\left(E_{L}=\right.$ $\left.2 J\left(1-\cos \left(\frac{P}{2}\right)\right)\right)$.

Equation (10) can be solved analytically in the thermodynamic limit $(N \rightarrow \infty)$ when $Q$ becomes a continuous variable ranging from 0 to $2 \pi$. In this limit the sum in (10) is replaced by an integral,

$$
1=\frac{1}{\pi} \int_{0}^{\pi} \mathrm{d} Q \frac{\cos (Q)\left[\cos \left(\frac{P}{2}\right)-\cos (Q)\right]}{E_{s} / 2 J-1+\cos \left(\frac{P}{2}\right) \cos (Q)} .
$$

It follows directly from (11) that the energy of the bound state is thus

$$
E_{s}=J \sin ^{2}\left(\frac{P}{2}\right)
$$

which coincides with Bethe's result $[5,6]$.

Some care should however be taken to consider the amplitude in the continuum limit

$$
a(X)=\frac{C}{N} \sum_{Q} \frac{\cos (Q) \cos (Q X)}{\left[1-E / 2 J-\cos \left(\frac{P}{2}\right) \cos Q\right]} .
$$

A straightforward implementation of the continuum limit as discussed above would lead to violation of the symmetry constraint (5b). In order to preserve this constraint under the analytic continuation, it is necessary to operate with the symmetrized quantity, $A_{s}$, which coincides with $a(X)$ at the discrete points:

$$
A_{s}(X)=\frac{1}{2}(a(X)+a(N-X)) .
$$

This results in replacing the sum in (13) by an integral and, respectively, $C$ by $\bar{C}$ :

$$
\begin{aligned}
A_{s}(X)= & -\frac{\bar{C}}{2} \int_{0}^{\pi} \frac{\cos (Q) \cos (Q X)}{\left[E_{s} / 2 J-1+\cos \left(\frac{P}{2}\right) \cos Q\right]} \frac{\mathrm{d} Q}{\pi} \\
& -\frac{\bar{C}}{2} \int_{0}^{\pi} \frac{\cos (Q) \cos Q(N-X)}{\left[E_{s} / 2 J-1+\cos \left(\frac{P}{2}\right) \cos Q\right]} \frac{\mathrm{d} Q}{\pi} .
\end{aligned}
$$

For integer $X>0$, one has (see, e.g., [11]):

$$
\begin{aligned}
& \int_{0}^{\pi} \frac{\cos (Q) \cos (Q X)}{[B-\cos Q]} \frac{\mathrm{d} Q}{\pi}=\frac{1}{2\left(B^{2}-1\right)^{1 / 2}} \\
& \quad \times\left[\left(B-\left(B^{2}-1\right)^{1 / 2}\right)^{X+1}+\left(B-\left(B^{2}-1\right)^{1 / 2}\right)^{X-1}\right],
\end{aligned}
$$

where $B \equiv \frac{1-\frac{1}{2} \sin ^{2}\left(\frac{P}{2}\right)}{\cos \left(\frac{P}{2}\right)}$. Then the symmetrized amplitude becomes

$$
\begin{aligned}
A_{s}(X) & =\frac{\bar{C}}{2 \sin ^{2}\left(\frac{P}{2}\right)}\left(\cos ^{2}\left(\frac{P}{2}\right)+1\right) \\
\times & {\left[\cos ^{X-1}\left(\frac{P}{2}\right)+\cos ^{N-X-1}\left(\frac{P}{2}\right)\right], X>0 . }
\end{aligned}
$$

The constant $\bar{C}$ is determined by the normalization condition

$$
1=\sum_{X=1}^{N-1} A_{s}^{2}(X)
$$

where the summation ranges over the physical states of the system. Taking (14) into account, one rewrites (15) as follows

$$
\begin{gathered}
1=\frac{\bar{C}^{2}}{4 \sin ^{4}\left(\frac{P}{2}\right)} \frac{\left(\cos ^{2}\left(\frac{P}{2}\right)+1\right)^{2}}{\cos ^{2}\left(\frac{P}{2}\right)} \\
\times \sum_{X=1}^{N-1}\left[\cos ^{2 N}\left(\frac{P}{2}\right)+\cos ^{2(N-X)}\left(\frac{P}{2}\right)+2 \cos ^{N}\left(\frac{P}{2}\right)\right] \\
=\frac{\bar{C}^{2}}{2 \sin ^{4}\left(\frac{P}{2}\right)} \frac{\left(\cos ^{2}\left(\frac{P}{2}\right)+1\right)^{2}}{\cos ^{2}\left(\frac{P}{2}\right)} \\
\times \frac{\cos ^{2}\left(\frac{P}{2}\right)+\cos ^{N}\left(\frac{P}{2}\right) \sin ^{2}\left(\frac{P}{2}\right)(N-1)-\cos ^{2 N}\left(\frac{P}{2}\right)}{\sin ^{2}\left(\frac{P}{2}\right)} \\
\simeq \bar{C}^{2} \frac{\left(\cos ^{2}\left(\frac{P}{2}\right)+1\right)^{2}}{2 \sin ^{6}\left(\frac{P}{2}\right)} .
\end{gathered}
$$

where exponentially small terms are neglected. Therefore, the amplitude of the even-parity bound state is

$$
\begin{aligned}
A_{s}(X)=\frac{1}{\sqrt{2}} & \sin \left(\frac{P}{2}\right) \\
& \times\left[\cos ^{X-1}\left(\frac{P}{2}\right)+\cos ^{N-X-1}\left(\frac{P}{2}\right)\right] .
\end{aligned}
$$


Let us now consider the antisymmetric solution. Proceeding in the same manner as for the even-parity amplitude, one obtains the Fourier transform of the master equation (4) which is identical to (9). A naive continuum limit solution of the compatibility equation gives then the same eigenenergy of the bound state (12). Within the continuum limit, the amplitude is obtained by taking the antisymmetrized combination

$$
A_{a}(X)=\frac{1}{2}(a(X)-a(N-X)) .
$$

After an analogous normalization procedure, it is explicitly expressed as

$$
\begin{aligned}
A_{a}(X)=\frac{1}{\sqrt{2}} & \sin \left(\frac{P}{2}\right) \\
& \times\left[\cos ^{X-1}\left(\frac{P}{2}\right)-\cos ^{N-X-1}\left(\frac{P}{2}\right)\right] .
\end{aligned}
$$

The orthogonality of the even and odd solutions on the physical interval $(X=1,2, \ldots, N-1)$ can be easily verified. Both solutions are defined on the interval $P \in[0,2 \pi)$ and are distributed in an interlaced order according to even or odd values of $l=N P / 2 \pi$. The total number of bound states is thus approximately equal to $N$. However, a closer look at equation (10) for the energy of the bound state shows that the even parity solution is stable for all the values of total momentum or, in other words, it does not cross the lower bound of the two magnon continuum. Indeed, the r.h.s. of equation (10) at the crossing point diverges due to the singularity at $Q=0$. This is actually the way which brings the even parity solution to be below the magnon continuum. On the contrary, due to the shifted sequence of allowed values of $Q$ in (8), the odd parity solution may cross the scattered magnon states at some critical total momentum $P=P_{\mathrm{c}}$. Indeed, one easily finds the exact value of $P_{\mathrm{c}}$ for the discrete chain because (10) directly transforms to

$$
1=\frac{1-\cos \left(\frac{P_{c}}{2}\right)}{N} \sum_{Q=\frac{\pi}{N}, \frac{3 \pi}{N} \ldots, 2 \pi-\frac{\pi}{N}} \frac{1}{(1-\cos Q)} .
$$

After summation in (18) (see, e.g. [11]), it gives an exact analytic relation

$$
\left(1-\cos \left(\frac{P_{\mathrm{c}}}{2}\right)\right)=\frac{2}{N}
$$

Therefore $P_{\mathrm{c}} \simeq \frac{4}{\sqrt{N}}$. It is interesting to note that this value of $P_{\mathrm{c}}$ is precisely the Bethe's estimate for a finite chain. It is also quite remarkable that the result in (19) actually $i s$ the exact lower boundary solution of Bethe's transcendental equation for the bound states. In this meaning it is "more exact" than the $P_{\mathrm{c}}$ value obtained from a $1 / N$ expansion [8]. We mention that (19) is also an outcome of problem (6) in reference [10], and can be derived by inspection of the respective Bethe equation

$$
\sinh (N v)\left(\cosh v-\cos \left(\frac{P}{2}\right)\right)=\sinh v(1+\cosh (N v))
$$

where $v=\mathrm{i}\left(k_{2}-k_{1}\right) \in \mathbf{R}$ and $k_{1}, k_{2}$ are Bethe quantum wavenumbers.

It can now be seen that in order to account for the long wavelength instability, the continuum limit must describe the finite size difference between both types of solutions. We note that the neighbourhood of $\sim \frac{\pi}{N}$ around the singular point $Q=0$ should be excluded from consideration since there are no physical states with $Q<\frac{\pi}{N}$ in the discrete counterpart. Therefore, the value of the shift mentioned above plays the role of a natural cutoff for the continuum limit. The singularity which keeps the evenparity solution away from the boundary of the scattered states disappears in the continuum limit of the instability condition and instead of (18), one arrives at the equation

$$
1=\frac{\left(1-\cos \left(\frac{P_{\mathrm{c}}}{2}\right)\right)}{\pi} \int_{\frac{\pi}{N}}^{\pi+\frac{\pi}{N}} \frac{\mathrm{d} Q}{(1-\cos (Q))} .
$$

The choice of the upper limit of integration is mainly motivated by the reasons of preserving the "volume" of the phase space as a result of introducing the cutoff at long wavelengths. The discrete states entering the summation in equation (18) can be divided in two equivalent parts connected by the relation $Q^{\prime}=2 \pi-Q$ and containing $N / 2$ states each (consider $N$ to be an even number ) and the sum can be restricted to half the number of discrete points after including the trivial factor 2. As the total volume of the phase space is $2 \pi$, we get an elementary "volume" $2 \pi / N$ corresponding to one state in the continuum representation. The elementary region of the first point at the lower boundary of integration thus covers the interval $[\pi / N, \pi / N+2 \pi / N)$. The total integration interval is formed by the union of $N / 2$ such intervals, i.e. $[\pi / N, \pi / N+2 \pi / N) \cup \ldots[\pi-\pi / N, \pi-\pi / N+2 \pi / N)=$ $[\pi / N, \pi+\pi / N)$ which is used in the equation above. Thus we have applied the shift to the whole interval and kept the shifted value of the upper limit which does not affect the low energy spectrum. Then we obtain the equation

$$
\left(1-\cos \left(\frac{P_{\mathrm{c}}}{2}\right)\right)=\frac{\pi}{2} \sin \left(\frac{\pi}{N}\right)
$$

It leads to the approximate continuum result: $P_{\mathrm{c}} \simeq \frac{2 \pi}{\sqrt{N}}$. Admittedly, some arbitrariness in the choice of integration limits can slightly modify the results of the approximation. For instance, dropping the $\pi / N$ shift of the upper limit does not change the above result, although it introduces a $1 / N$ correction to the r.h.s. of (19). However it can be easily checked that the continuum version of the eigenenergy equation (10) with our choice of the upper limit reproduces Bethe's exact result for $P$ at the boundary of the Brillouin zone $\left(E_{a}=J\right)$, while the omission of the $\pi / N$ shift at the upper boundary yields a slightly higher energy.

We recall that in the exact solution the energy of the odd parity bound state $E_{a}$ is higher than that of the even parity state (12). The same follows from our approach due to the shifted limits of integration compared to (11). After 
integration we get:

$$
1+\frac{2}{\pi} \frac{D}{G}=-\frac{2}{\pi} \frac{\left(G+\cos \left(\frac{P}{2}\right)\right)}{\sqrt{\left(G^{2}-1\right)}} \arctan \left(\frac{\sqrt{\left(G^{2}-1\right)}}{D}\right)
$$

where $G=\frac{E_{a} / 2 J-1}{\cos \left(\frac{P}{2}\right)}$ and $D=\sin \left(\frac{\pi}{N}\right)$.

It follows from (21) that the odd-parity bound state lies above the even-parity one and their separation grows as the wavelength increases. At the boundary of the Brillouin zone both states have the same energy $J$. Beyond the critical momentum (20), for large $N$, we can apply the $1 / N$ expansion in (21). This results in the correction for the bound state energy for $P \gg P_{\mathrm{c}}$ :

$$
E_{a} \simeq J \sin ^{2}\left(\frac{P}{2}\right)\left(1+\frac{4}{N} \frac{\cos \left(\frac{P}{2}\right)}{\left(1+\cos ^{2}\left(\frac{P}{2}\right)\right)}\right) .
$$

Comparison with the respective correction following from the Bethe ansatz

$$
E_{a, s} \simeq J \sin ^{2} \frac{P}{2}\left(1 \pm \sin ^{2} \frac{P}{2} \cos ^{N-2} \frac{P}{2}\right)
$$

where the +/ - signs correspond to odd and even solutions respectively, shows that our approximation gives a power law correction while the exact solution predicts an exponential one. This is a consequence of the continuum approximation which is naturally expected to be inferior at large momenta as compared to small $P$. Indeed, if we now determine the expansion corresponding to the region close to the critical momentum $\left(P-P_{\mathrm{c}} \ll P_{\mathrm{c}}\right)$ we find the same behaviour for both the exact solution and our approximation with the respective values of $P_{\mathrm{c}}$ :

$$
\frac{E_{a}}{J} \simeq 2\left(1-\cos \left(\frac{P}{2}\right)\right)-\frac{3 P_{\mathrm{c}}^{2}}{2 N}\left(\frac{P}{P_{\mathrm{c}}}-1\right) .
$$

Exactly as for the Bethe ansatz no bound states are possible at $P<P_{\mathrm{c}}$ : the equation (21) has no real odd parity solutions since the quantity $G^{2}<1(G<0)$. Instead there exist plane wave two magnon states. The excitation spectrum of the odd-parity bound state is illustrated in Figure 1 where the size dependent features are emphasized by taking a small number of spins $N=20$. Note that all the energies displayed in the figure are smooth functions of total momentum. For comparison with the exact solutions of the Bethe Ansatz, we must evaluate these functions at the discrete points, corresponding to the allowed momenta for the finite chain. For the even solutions the two results coincide with exponential accuracy as noted above (Eqs. (12) and (23)). For the odd solutions a detailed numerical comparison is presented in Table 1.

In order to clarify the physical meaning of the instability, it is worth noticing that within the present approach, the term on the r.h.s. of the equation (4) does not play the role of a boundary condition for free magnons,

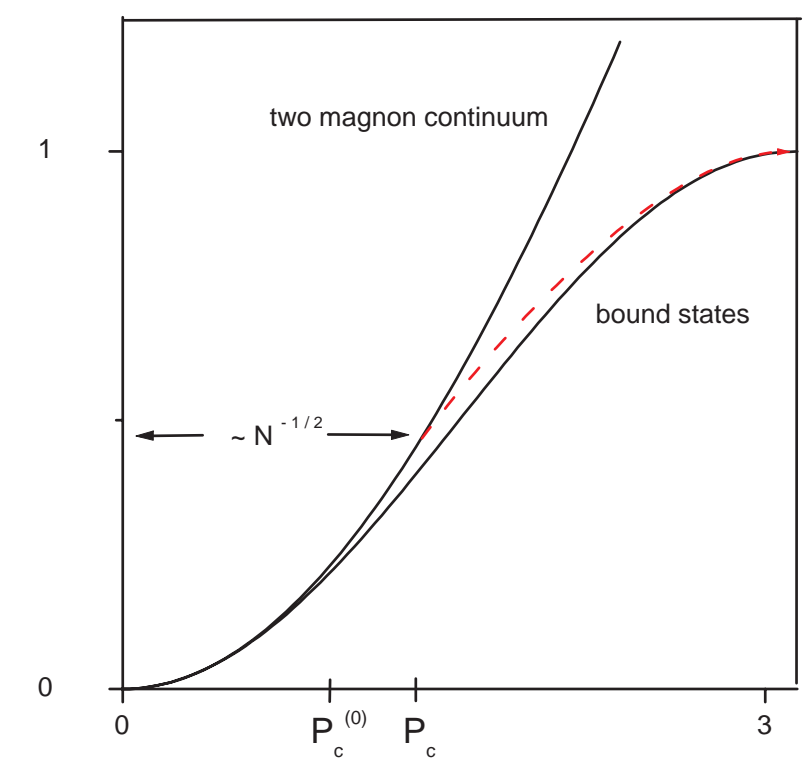

Fig. 1. The spectrum of the odd-parity bound state in (21) (dashed line) has a higher energy than the even-parity one (12) (solid line) and decays into scattered magnon states at $P_{\mathrm{c}} \simeq \frac{2 \pi}{\sqrt{N}}, N=20,($ Eq. $(20))$. The value of $P_{\mathrm{c}}$ is close to the exact one $P_{\mathrm{c}}^{(0)}$ defined by (19).

Table 1. Energy of the odd parity solutions in the discrete points for $N=20$. The value $E_{a}^{\text {exact }}$ is obtained from Bethe's ansatz and $E_{a}$ is determined by the equation (21). The critical momentum for the exact solution is $P_{\mathrm{c}}^{(0)}=0.894$ and for the approximate solution $P_{\mathrm{c}}=1.4$. Therefore the smallest allowed values of $l$ are 3 and 5 respectively.

\begin{tabular}{|l|l|l|l|l|}
\hline$P=\frac{2 \pi}{N} l, l=3,5,7,9$ & 0.942 & 1.571 & 2.199 & 2.827 \\
\hline$E_{a} / J$ & - & 0.550 & 0.862 & 0.999 \\
\hline$E_{a}^{\text {exact }} / J$ & 0.215 & 0.500 & 0.794 & 0.976 \\
\hline$E_{L} / J$ & 0.218 & 0.586 & 1.092 & 1.687 \\
\hline
\end{tabular}

as in Bethe's ansatz. In other words it does not vanish exactly. This term actually describes the magnon interaction. As shown below, its vanishing at the critical point is the actual origin of instability. Indeed, substituting $E_{L}(P)=2 J\left(1-\cos \left(\frac{P}{2}\right)\right)$ into (13) for the odd-parity amplitude and summing over the range (8) leads to

$$
a(X)=U\left(\frac{N}{2}-X\right)
$$

The constant $U$ depends only on $N$, viz., $U=\frac{\sqrt{24}}{N^{3 / 2}}+$ $O\left(N^{-5 / 2}\right)$. The formula (24) is valid for $X \geq 1$, i.e., within the physical range of relative separation. However, as discussed in references [4] and [6], equation (4) defines the amplitude at $X=0$ and in fact, this definition should be based on the arguments of continuity of the wave function. Therefore by the continuity argument we can extend (24) to the whole range of $X$. Thus, as the energy of the bound state crosses the lower boundary of the magnon 


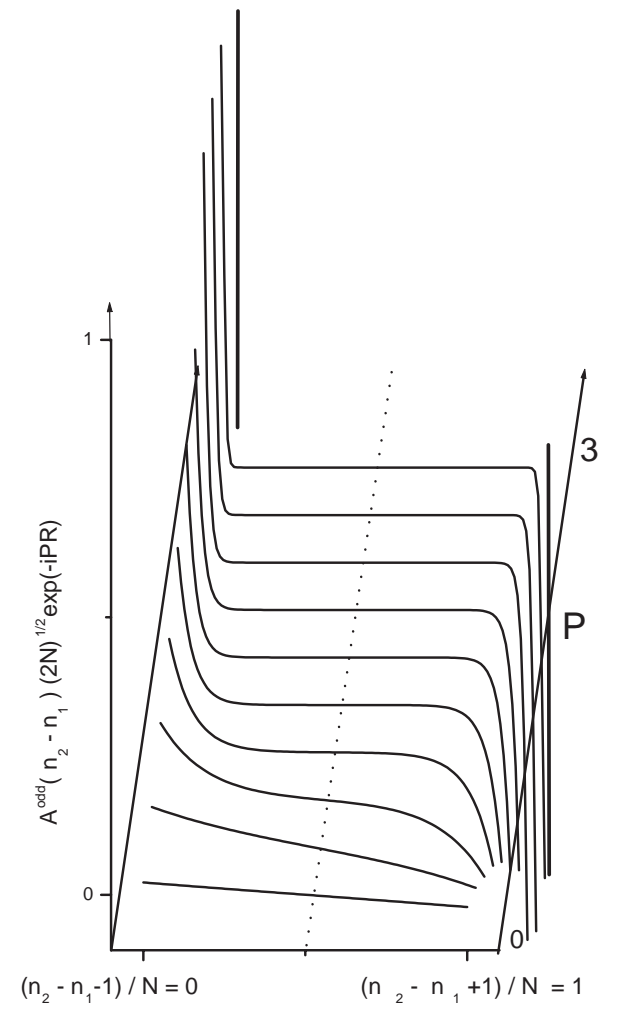

Fig. 2. The continuum amplitude of the-odd parity bound state as a function of distance between overturned spins for $N=100$ in the physical region: $1-\frac{1}{N} \leq \frac{n_{2}-n_{1}}{N} \leq \frac{1}{N}$. Total momentum $P$ of the excitation is equal to $\frac{2 \pi}{N} l$ where odd integer $l$ ranges in the interval $[0, N-1]$. Extrema are located at the edges of the interval, i.e., at positions of the nearest neighbours.

continuum, the amplitude becomes linear in the relative separation between spin deviations. Then the bracketed term in (4) becomes:

$$
\begin{aligned}
\cos \left(\frac{P}{2}\right) a(0)-a(1) & =U\left[\cos \left(\frac{P}{2}\right) \frac{N}{2}-\left(\frac{N}{2}-1\right)\right] \\
& =U\left(1-\frac{N}{2}\left[1-\cos \left(\frac{P}{2}\right)\right]\right) .
\end{aligned}
$$

One can see that the magnon interaction becomes equal to zero precisely at the critical point (19). This means that the bound state decays simply due to vanishing of interaction at $P_{\mathrm{c}}$ as a consequence of the linear spatial dependence of the amplitude. It can be also demonstrated that the continuum approximation agrees with the above arguments. For instance, Figure 2 displays the evolution of the odd-parity amplitude (17) over the Brillouin zone. This amplitude becomes linear in the long wavelength region whereas the even parity one (Fig. 3) remains stable due to retaining its nonlinear solitonic form for all values of the total momentum. Particularly, at small $P$, it shows a quadratic dependence.

A direct comparison shows that the amplitudes in the continuum limit coincide with Bethe's exact solution as

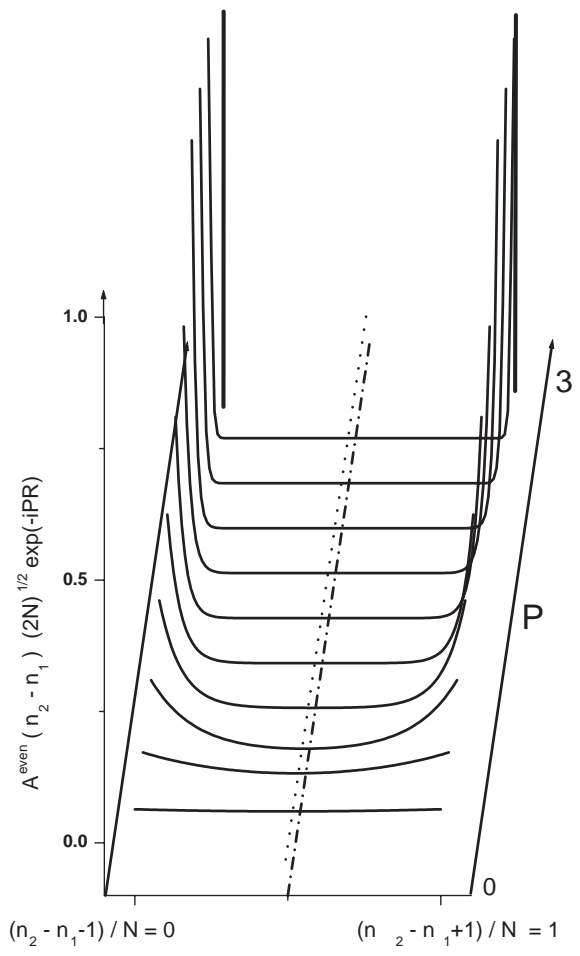

Fig. 3. The continuum amplitude of the even-parity bound state as a function of distance between overturned spins. Total momentum $P$ of the excitation is equal to $\frac{2 \pi}{N} l$ where integer $l$ takes even numbers from the interval $[0, N-1]$. The present result coincides with the exact one obtained in [8] via using Bethe's ansatz.

$N \rightarrow \infty$. For instance, at $P=\pi-0^{+}$, Bethe's normalized solution gives rise to a bound state with two overturned spins located precisely at the neighbour sites $[9,10]$ :

$$
\begin{aligned}
& a(X)=\frac{1}{\sqrt{2}}[\exp (-v(X-1)) \\
& \pm \exp (-v(N-X-1))]_{v \longrightarrow \infty}=\frac{1}{\sqrt{2}}\left(\delta_{X, 1} \pm \delta_{X, N-1}\right) .
\end{aligned}
$$

Here

$$
v=-\ln \left(\cos \left(\frac{P}{2}\right)\right), X=1, \ldots, N-1
$$

Interestingly, the same strictly Kronecker-type solitonic behaviour results from our equations (16) and (17) since $\cos ^{n}\left(\frac{\pi}{2}-0^{+}\right)=\sin ^{n}\left(0^{+}\right)=\delta_{n, 0}$. This solution corresponds to the state with two spin deviations at the nearest-neighbour sites propagating along the chain as a single object without any internal dispersion. For smaller momenta, the amplitude $a(X)$ spreads over the lattice, although its maximum is always located at the nearestneighbour position. For example, in the neighbourhood of 
$P=\pi$, one introduces $\tilde{P}=\pi-P \ll 1$ and obtains then

$$
\begin{aligned}
A(X) \simeq & \frac{\cos \left(\frac{\tilde{P}}{2}\right)}{\sqrt{2}}[\exp (-(X-1)|\ln (\tilde{P})|) \\
& \pm \exp (-(N-X-1)|\ln (\tilde{P})|)]
\end{aligned}
$$

In the opposite limit of long wave excitation $\left(\frac{1}{\sqrt{N}}<P \ll\right.$ $1, X \ll N / 2$ ), one easily derives that

$$
A(X) \simeq \frac{\sin \left(\frac{P}{2}\right)}{\sqrt{2}} \exp \left(-(X-1) \frac{P^{2}}{8}\right) .
$$

It is worth mentioning that these results agree with Bethe's ansatz only in the physical region but not outside it $(X<1$ and $X>N-1)$ where Bethe's solution requires $\cos \left(\frac{P}{2}\right) a(0)-a(1)=0$. For instance, at $P=\pi$, this implies $a(0)=\infty$, whereas our result is finite.

\section{Long wavelength expansion}

In the previous section we have performed the continuation to infinity in the reciprocal Fourier space via replacing finite sums by integrals and keeping track of the discrete $N$ values in the integration limits. The present section deals with an alternative differential approach derived from the master equation by introducing the continuum limit in direct space. This is achieved by defining the length scale parameter, namely, the lattice spacing $c=L / N$ where $L$ is the length of the chain. The continuum limit means then that $N \rightarrow \infty, c \rightarrow 0$, while $L$ is kept constant and the following correspondence applies: $\sum_{X} \rightarrow \frac{1}{c} \int_{0}^{L} \mathrm{~d} x, \sum_{P} \rightarrow$ $\int_{0}^{2 \pi} \mathrm{d}(p c)$ and $\delta_{X, 1} \rightarrow c \delta(x-c)$ where $x=X c$ and $p=\frac{P}{c}$. Equation (4) is thus transformed to the following differential equation:

$$
\begin{aligned}
& {\left[E-2 J+2 J \cos \left(\frac{p c}{2}\right) \cos \left(-\mathrm{i} c \frac{\partial}{\partial x}\right)\right] a(x)=} \\
& \quad J c\left[\cos \left(\frac{p x}{2}\right) a(0)-a(x)\right][\delta(x-c)+\delta(x+c)] .
\end{aligned}
$$

It is worth mentioning that the wave phase which bears a direct physical meaning remains unchanged under transform $P \Delta=p c$ where $\Delta$ is an integer $(\Delta=1$ for the nearest neighbours). For instance, $\Delta$ determines the angle between the neighbouring spins participating in the spin wave excitation. It can be treated as an expansion parameter in solving equation (26).

It is of interest to study a variety of approximations introduced by truncating the free magnon dispersion due to their significance in applications or when no exact expression for the one-magnon spectrum is known. Taking a long wavelength expansion in powers of $(p c)$ and $\left(-\mathrm{i} c \frac{\partial}{\partial x}\right)$ in the 1.h.s. of (26) which describes the free magnon dispersion, one obtains the quadratic approximation

$$
\begin{aligned}
& {\left[E / 2 J-1+\left(1-\frac{p^{2} c^{2}}{8}+\frac{c^{2}}{2} \frac{\partial^{2}}{\partial x^{2}}\right)\right] a(x)=} \\
& \frac{c}{2}\left[a(0) \cos \left(\frac{p x}{2}\right)-a(x)\right][\delta(x-c)+\delta(x+c)] .
\end{aligned}
$$

For the even-parity solution, the eigenenergy is determined by the equation

$$
1=-\frac{2}{\pi} \int_{0}^{\pi} \frac{\cos (q c)\left[\cos \left(\frac{p c}{2}\right)-\cos (q c)\right]}{\left(D+(q c)^{2}\right)} \mathrm{d}(q c),
$$

where $D \equiv\left(\frac{p c}{2}\right)^{2}-E / J$ should be positive to support the existence of a bound state. Then, in the long wave limit, (27) yields a bound state with the energy $E_{1}$ which lies slightly below the lower boundary of the two-magnon continuum of the exact solution: $E_{L}-E_{1}=J\left(\frac{p c}{4}\right)^{4}, p c \ll 1$, while $E_{L}-E_{s}=\frac{J}{4}\left(\frac{p c}{2}\right)^{4}, p c \ll 1$. However, such solution is not physical because it crosses $E_{L}$ at some finite value of $(p c)$. This implies that a quadratic approximation is insufficient to describe the bound state even qualitatively, as anticipated, since the energy of the exact ground state peals off from the continuum in the 4 th order in $P$.

One may therefore take higher-order terms in the free magnon dispersion to improve the quadratic approximation and, indeed, obtain both types of bound states within the fourth-order approximation to the free magnon dispersion. However, one should remind that the factor $\cos \left(\frac{p c}{2}\right)$ in the l.h.s. of (26) originates from an explicit use of the translational symmetry in deriving equation (3). Thus as far as the translational motion is taken precisely into account, any long wavelength approximation can be considered only for the relative motion. These approximations are described as a series expansion in the powers of $\left(-\mathrm{i} c \frac{\partial}{\partial x}\right)$. The simplest one derived in such a manner is the following second-order differential equation

$$
\begin{gathered}
{\left[E-2 J\left(1-\cos \left(\frac{p c}{2}\right)\right)+J \cos \left(\frac{p c}{2}\right) c^{2} \frac{\partial^{2}}{\partial x^{2}}\right] a(x)=} \\
J c\left[\cos \left(\frac{p x}{2}\right) a(0)-a(x)\right][\delta(x-c)+\delta(x+c)] .
\end{gathered}
$$

We show that the solution of equation (28) describes the bound state lying below the true two-magnon continuum and actually is very close to the exact solution in the whole interval of $(p c)$.

In the long-wave limit $((p c) \ll 1)$, the approximate bound state energy, $\tilde{E}_{s}$, is $\tilde{E}_{s} / 2 J-2 \sin ^{2}\left(\frac{p c}{4}\right) \simeq$ $-1.57 \sin ^{4}\left(\frac{p c}{4}\right) \cos \left(\frac{p c}{2}\right)$, while the exact solution can be cast in the form $E_{s} / 2 J-2 \sin ^{2}\left(\frac{p c}{4}\right)=-2 \sin ^{4}\left(\frac{p c}{4}\right)$. The long wave amplitude becomes very close to the exact one as well. For example, for $X \ll N / 2$, one obtains: $a(X)=0.334(p c) \exp \left(-0.124(p c)^{2}\left(\frac{x}{c}-1\right)\right)$. Notice that the approximate solution coincides with the exact one $\left(E_{s}=J\right)$ at the boundary of the Brillouin zone. 
We now demonstrate that the long-wave approximation is capable to adequately describe the aforementioned instability. Let us first determine the crossing point for the finite chain solution corresponding to the truncated magnon dispersion. By analogy with (18), one has

$$
1=\frac{2}{N \cos \left(\frac{P_{\mathrm{c}}}{2}\right)} \sum_{Q=\frac{\pi}{N}, \ldots, 2 \pi-\frac{\pi}{N}} \frac{\cos (Q)\left(\cos Q-\cos \left(\frac{P_{\mathrm{c}}}{2}\right)\right)}{Q^{2}} .
$$

Its numerical solution gives $P_{\mathrm{c}} \simeq \frac{5.66}{\sqrt{N}}$, while its continuum counterpart is $p_{\mathrm{c}} \simeq \frac{6.68}{c \sqrt{N}}$. Comparison of these values with the corresponding one in (19) and (20) obtained without truncation shows a remarkably good agreement. One may also figure out that the truncation tends to increase the instability region and such tendency has a clear physical explanation. The physics behind the contrasting long wave behaviour of the two types of bound excitations is similar to that of bonding and antibonding electron states. As is illustrated by Figures 2 and 3, the difference in the absolute values of both amplitudes is the largest at large separations between the flipped spins. Thus, spins in an odd-parity state are bound more loosely than in the evenparity one. Such difference becomes more pronounced as the total momentum decreases. For a long-wave excitation, the flipped spins are distributed over a larger distance covering the region of the depleted odd parity amplitude, the "weak link" of the collective excitation. If the magnons are stiff enough, they are able to destroy such a bound state. Since the approximations discussed above in connection with equation (28) consist in replacing the true magnon dispersion by a more stiff dependence, a disruption of the pair at shorter wavelengths becomes possible.

Altogether with the energy spectrum illustrated by Figure 4, one may conclude that the truncation approximation is rather close to the exact result and the reason for this lies actually in the behaviour of the amplitude studied in the present paper. Indeed, we show that spin deviations in the bound state are most likely to occur at the nearestneighbour sites. In other words, the two magnons move throughout the lattice with essentially the same velocity and the shape of this wave is rather smooth, or, in other words, the dispersion of the relative motion in the bound state is small and its linearized treatment is thus fully justified. On the contrary, the states corresponding to the separated magnons are substantially delocalized over the whole lattice and, therefore, their amplitudes are localized in the momentum space. In fact, for a given total momentum, such values of the relative momentum can be used to parametrize the continuum of scattered magnon states. Hence, one may predict that for scattered states, the longwavelength approximation would give rather poor results. This is easily seen by considering the projection of the single magnon state onto the subspace discussed in the present work. As mentioned in Section 2, its exact amplitude is $\cos \left(\frac{P X}{2}\right)$ and this ensures that such state is interaction free for all momenta. Then, by introducing the exact amplitude in the approximate equation (28), one obtains its energy $E_{1}^{t} / J=4 \sin ^{2}\left(\frac{P}{4}\right)-\frac{P^{2}}{4} \cos \left(\frac{P}{2}\right)$. It ob-

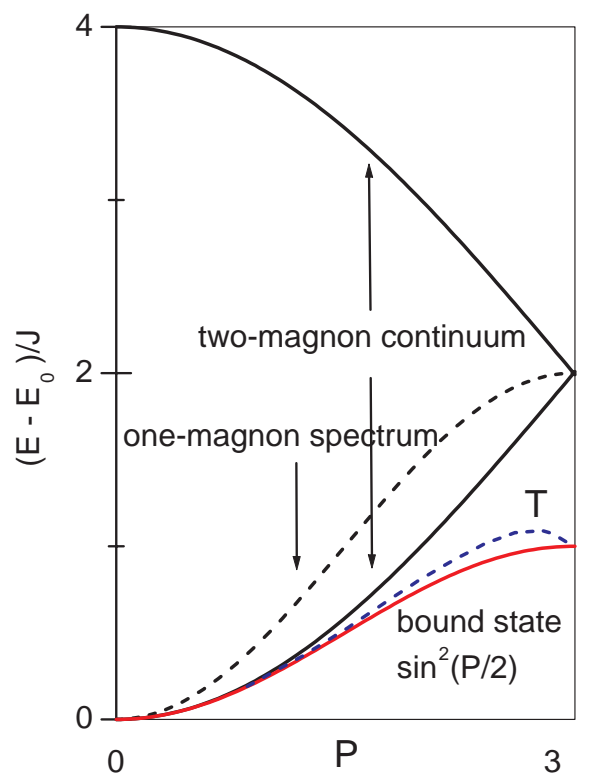

Fig. 4. Spectrum of the two-magnon excitations within the continuum limit approach for $N \rightarrow \infty$. The bound state solution of the truncated master equation (27) is indicated by letter T. This approximation is rather close to the exact one for the entire Brillouin zone, and for finite $N$ it reproduces well the critical point of the odd-parity bound state (28).

viously provides a poor estimate because it is even lower than the energy of the bound state.

\section{Conclusions}

By means of a further development of the continuum approach [4-6], we have obtained the amplitude of the twomagnon bound state for the $1 \mathrm{D}$ ferromagnetic $S=1 / 2$ Heisenberg model. It coincides with Bethe's exact solution in the thermodynamic limit. The agreement of the present approach with the exact solution for a finite chain encourages further application of this approach to mesoscopic systems. The continuation procedure is based on the explicit inclusion of the translational symmetry into the amplitude. This allows us to obtain for the first time the orthonormalized continuous forms of the two types of solution with even and odd parities as $N \rightarrow \infty$.

We have also shown that the present approach adequately describes the contrasting behaviour of the two types of bound long wavelength magnon excitations. The instability of the odd-parity bound state occurs at rather long wavelengths, of the order of $1 / \sqrt{N}$. The physics of this instability is related to vanishing of the magnon interaction which remains hidden within Bethe's ansatz treating it as the boundary condition. While the even parity bound state retains its nonlinear solitonic-type shape for all values of the total momentum of the excitation, the odd-parity one acquires a linear shape throughout the whole lattice at the critical point where it dissociates into free magnons. 
In view of further applications of the present continuum limit approach to more complicated geometries, we have analysed different long-wave approximations. We have solved the truncated version of the exact differential equation describing the continuum limit in $1 \mathrm{D}$ and estimated its deviation from the exact solution due to linearizing the magnon dispersion. As anticipated, the quadratic truncation fails to describe a bound state. Its further improvement which takes the translational symmetry into account provides a good agreement with the complete solution over the whole Brillouin zone, even including the size dependent instability mentioned above (see, e.g., Fig. 3). The fact that such approximation turns out to do so well demonstrates that the formation of a bound excitation requires the magnons to move with nearly the same velocity. The longer the total wavelength of the excitation, the larger is the dispersion, and the weaker the bound state.

Financial support from the Concerted Action Scheme of the Flemish Government and from the National Science Foundation (FWO) is gratefully acknowledged. One of us, S.C., also acknowledges support from the Supreme Council for Scientific and Technological Development of Moldova and thanks the Abdus Salam ICTP for hospitality.

\section{References}

1. A. Ceulemans, I. Vos, Mol. Phys. 72, 1051 (1991).

2. A. Ceulemans, P.W. Fowler, I. Vos, J. Chem. Phys. 100, 549 (1994)

3. E. Fradkin, Field Theories of Condensed Matter Systems (Redwood City, CA., Addison Wesley, 1991), 350 pp.

4. N. Fukuda, M. Wortis, J. Phys. Chem. Solids 24, 1675 (1963).

5. M. Wortis, Phys. Rev. 132, 85 (1963).

6. D.C. Mattis, The Theory of Magnetism, Vol. I (Springer Verlag, Berlin, Heidelberg, 1981), 300 pp.

7. Yu.A. Izyumov, M.I. Katsnelson, Yu.N. Skriabin, Statistical Mechanics of Magnetically Ordered Systems (New York, NY., Consultants Bureau, 1988), 295 pp.

8. H. Bethe, Z. Phys. 71, 205 (1931).

9. T. Lulek, in Properties of Condensed Matter, edited by T. Lulek, B. Lulek, A. Wal (World Scientific, Singapore, 1998), p. 52.

10. M. Karbach, G. Müller, Comput. Phys. 11, 36 (1997).

11. I.S. Gradshtejn, I.M. Ryzhik, A. Jeffrey, Table of Integrals, Series, and Products, 5th edn. (New York, NY., Academic Press, 1993), 1204 pp. 